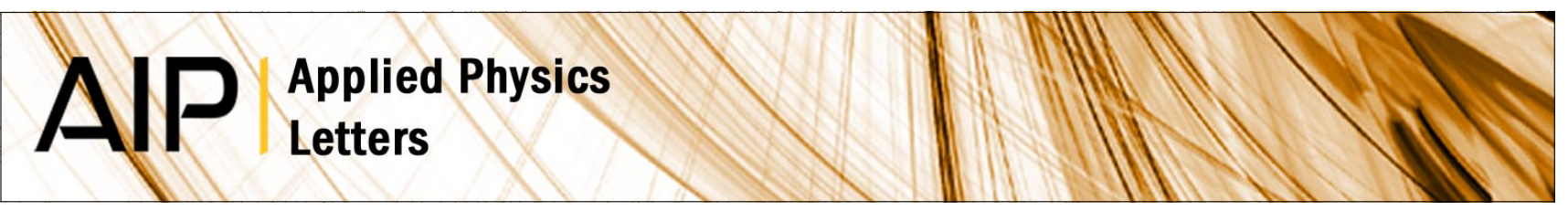

\title{
Shear-band arrest and stress overshoots during inhomogeneous flow in a metallic glass
}

R. Maaß, D. Klaumünzer, G. Villard, P. M. Derlet, and J. F. Löffler

Citation: Appl. Phys. Lett. 100, 071904 (2012); doi: 10.1063/1.3684871

View online: http://dx.doi.org/10.1063/1.3684871

View Table of Contents: http://apl.aip.org/resource/1/APPLAB/v100/i7

Published by the American Institute of Physics.

\section{Related Articles}

Generalized Flory-Huggins theory-based equation of state for ring and chain fluids

J. Chem. Phys. 136, 124904 (2012)

The plastic and liquid phases of $\mathrm{CCl} 3 \mathrm{Br}$ studied by molecular dynamics simulations

J. Chem. Phys. 136, 094515 (2012)

Rheological, optical, and thermal characterization of temperature-induced transitions in liquid crystal ferrosuspensions

J. Appl. Phys. 111, $07 B 308$ (2012)

Comment on "Design of acoustic devices with isotropic material via conformal transformation" [Appl. Phys. Lett. 97, 044101 (2010)]

Appl. Phys. Lett. 100, 066101 (2012)

The role of the isothermal bulk modulus in the molecular dynamics of super-cooled liquids

J. Chem. Phys. 135, 244508 (2011)

\section{Additional information on Appl. Phys. Lett.}

Journal Homepage: http://apl.aip.org/

Journal Information: http://apl.aip.org/about/about_the_journal

Top downloads: http://apl.aip.org/features/most_downloaded

Information for Authors: http://apl.aip.org/authors

\section{ADVERTISEMENT}

\section{(e) ACCELERATE AMBER AND NAMD BY 5X. NVIDIA TRYIT ON A FREE, REMOTELYYHOSTED CLUSTER.}




\title{
Shear-band arrest and stress overshoots during inhomogeneous flow in a metallic glass
}

\author{
R. Maaß, ${ }^{1, a), b)}$ D. Klaumünzer, ${ }^{1}$ G. Villard, ${ }^{1}$ P. M. Derlet,${ }^{2}$ and J. F. Löffler ${ }^{1}$ \\ ${ }^{1}$ Laboratory of Metal Physics and Technology, Department of Materials, ETH Zurich, Wolfgang-Pauli-Strasse 10, \\ 8093 Zurich, Switzerland \\ ${ }^{2}$ Condensed Matter Theory, Paul Scherrer Institut, 5232 Villigen PSI, Switzerland
}

(Received 16 December 2011; accepted 26 January 2012; published online 13 February 2012)

\begin{abstract}
At the transition from a static to a dynamic deformation regime of a shear band in bulk metallic glasses, stress transients in terms of overshoots are observed. We interpret this phenomenon with a repeated shear-melting transition and are able to access a characteristic time for a liquidlike to solidlike transition in the shear band as a function of temperature, enabling us to understand why shear bands arrest during inhomogenous serrated flow in bulk metallic glasses. (C) 2012 American Institute of Physics. [doi:10.1063/1.3684871]
\end{abstract}

Slowly cooling a liquid metal to ambient temperature is known to result in a phase transition from a liquid to a crystalline solid; a method that forms the basis for metals production, near net-shape casting, but also microstructural design when the cooling rate is varied. Most of our metals in daily use are crystalline, but pioneering work by Klement et al. ${ }^{1}$ demonstrated that very high cooling rates can quench the liquid metal to an amorphous solid, called a metallic glass. Subsequent progress in casting processes led to glassy structures with thicknesses of up to a centimeter, called bulk metallic glasses (BMGs)..$^{2-4}$

Since then, the understanding of plastic flow in BMGs both at elevated and low homologous temperatures has been a topic of intense research. ${ }^{5-7}$ Studies dedicated to hightemperature deformation have unveiled a Newtonian or nonNewtonian behaviour, where plastic flow at moderate strain rates is homogenously distributed throughout the sample. ${ }^{8,9}$ Decreasing the temperature to the undercooled liquid region and subsequently beyond the glass transition, the metal liquid experiences a tremendous increase in viscosity, finally entering the regime of an amorphous solid. Thus, BMGs can be viewed as a frozen liquid. ${ }^{7}$ In this regime, flow becomes inhomogeneous and drastically confined to nm-thick shear bands. ${ }^{5,7}$ Inhomogeneous flow either proceeds in a continuous non-serrated fashion, where shear-band propagation is driven by the testing device, or as serrated intermittent flow, when the shear-band propagation is faster than the applied strain rate. ${ }^{10,11}$ During serrated flow, shear-band propagation advances in a motion typical for that of stick-slip systems. ${ }^{12,13}$

In search for engineering applications, as, for example, structural components subjected to stresses passing the elastic limit, intense efforts have been undertaken to increase the plastic straining capability via microstructural design. ${ }^{14}$ Another route intensely explored is investigating the underlying physics on how plastic flow develops at the microstructural or atomic scale, ${ }^{5-7,15}$ which, however, entails severe experimental acces-

\footnotetext{
a) Author to whom correspondence should be addressed. Electronic mail: maass@caltech.edu.

b) Present address: Division of Engineering and Applied Sciences, California Institute of Technology, 1200 E. California Blvd., MC 309-81, Pasadena, California 91125-8100, USA.
}

sibility complications due to flow's confinement to the nmlength scale and rapid shear-band propagation during serrated flow that occurs within milliseconds at ambient temperature. ${ }^{10,11,16}$ Nevertheless, specialized experimental techniques applied after deformation suggest that the deforming material within a shear band undergoes a structural change towards lower density, ${ }^{17,18}$ being analogous to shear-induced dilation processes known to occur in a wide range of disordered or granular media. ${ }^{19,20}$ This naturally emerges towards a picture in which the material in an active shear band is believed to be in a state of lower viscosity relative to the surrounding bulk matrix. Viscosity estimates derived from shear rates within the shear band assuming Couette flow ${ }^{21}$ and in-situ acoustic emission experiments ${ }^{22}$ are in agreement with this, yielding in the former case viscosity values close to those measured at the glass transition and in the latter case volume expansions during shear-band initiation equivalent to those measured in the undercooled liquid regime. Ultrahigh strain rate molecular dynamics simulations also indicate this picture, deriving that the initiation of flow can be viewed as a stress-induced glass transition confined to the shear band. ${ }^{23}$ Despite a lack of direct experimental evidence, there exists thus general consensus on shear-band initiation caused by localized structural softening due to a volume expansion, followed by shear-band propagation on a confined layer with reduced viscosity. Conversely, it remains an open question as to what the mechanisms are that govern the reverse transition to flow initiation, that is, shear-band arrest.

In this letter, we report on stress overshoots during inhomogeneous flow obtained from stop-and-start experiments. Whilst this work directly reveals shear-band creep, as well as static aging after shear-band arrest, we also observe a pronounced time dependence of the stress-overshoot magnitude. From analogous stop-and-start experiments on confined nanoscopic liquids, we relate the stress-overshoot magnitude to a characteristic nucleation time of a liquidlike to solidlike transition, ${ }^{24-27}$ and propose that this governs shear-band arrest. Such an approach may provide the key ingredients to fundamentally understanding the stable plastic flow of metallic glasses.

Stop-and-start experiments within the flow regime of a Zr-based $\left(\mathrm{Zr}_{52.2} \mathrm{Ti}_{5} \mathrm{Cu}_{17.9} \mathrm{Ni}_{14.6} \mathrm{Al}_{10}\right)$ BMG were conducted with a screw-driven compression machine at temperatures 

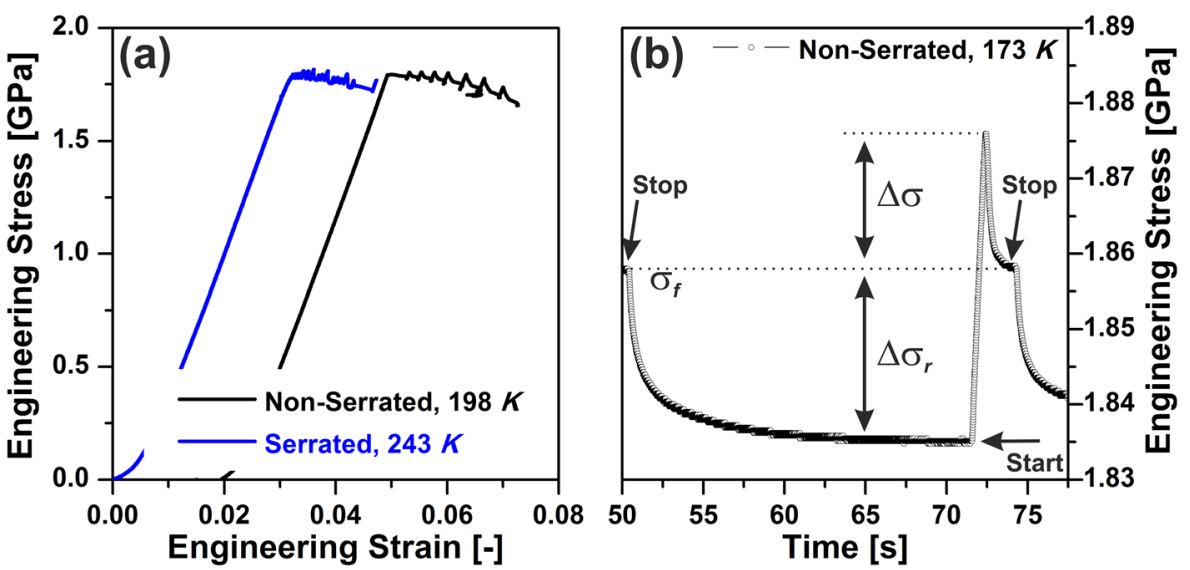

FIG. 1. (Color online) (a) Stress-strain response for stop-and-start experiments under serrated and non-serrated conditions, displaying stress overshoots upon resumed loading. A stress overshoot $\Delta \sigma$ following the restart of loading under non-serrated conditions is shown in (b); $\sigma_{f}$ indicates the flow stress at "stop" and $\Delta \sigma_{r}$ the stress relaxation occurring after stopping. between $78 \mathrm{~K}$ and $298 \mathrm{~K}$, at an applied strain rate $\dot{\varepsilon}$ of $10^{-3}$ $\mathrm{s}^{-1}$. Compression testing was performed in an experimental chamber with liquid nitrogen used as coolant. Further details on the setup can be found elsewhere. ${ }^{11,21}$ All samples were prepared from the same master alloy by suction casting of $3 \mathrm{~mm}$ rods in an arc-melter. The compression samples were $5 \mathrm{~mm}$ long and carefully polished on both ends to assure plane-parallel testing.

Figure 1(a) displays two stress-strain curves, one of which was tested under serrated $(243 \mathrm{~K})$ and the other under non-serrated $(198 \mathrm{~K})$ conditions. During both experiments, the drive of the machine was stopped ("stop," cross-head velocity $v_{X H}=0$ ) at a flow stress $\sigma_{f}$, and restarted ("start," $v_{X H} \neq 0$ ) several times within the plastic flow regime, yielding stress overshoots upon restarting. Such overshoots are well-known for homogeneous deformation of BMGs at high temperatures ${ }^{28}$ and have been recently reported on during inhomogeneous flow under cryogenic conditions. ${ }^{29}$ Figure 1(b) shows the stress-time response for a stop-and-start cycle recorded at $173 \mathrm{~K}$ during non-serrated flow, which upon stopping reveals a time dependent stress relaxation $\Delta \sigma_{r}$ and a stress-overshoot $\Delta \sigma$ before flow continues as prior to the stop. We define the time between "stop" and "start" as the stopping time or aging time, which will be shown to correlate with the overshoot magnitude $\Delta \sigma$.

Focusing first on the event of stopping within the flow regime at any flow stress $\sigma_{f}$, the subsequent stress-relaxation $\Delta \sigma_{r}$ as a function of time and temperature shows quickly ceasing resolvable stress decays at higher temperatures, whereas longer retaining decays are present at lower temperatures (Fig. 2). More importantly, below $123 \mathrm{~K}$, there exist vanishingly small differences in the displayed stress relaxation, as revealed by plotting the relaxation stress magnitude $\left|\Delta \sigma_{r}\right|$ at an arbitrary time $t_{r}$ of $2.6 \mathrm{~s}$ as a function of temperature (see inset of Fig. 2). Entering the stop-phase of the experiment from a regime of shear-band propagation leads to the conclusion that the stress-relaxation phenomena observed in Fig. 2 may be partly linked to the duration of reaching shear-band arrest under the conditions prevailing. Thus, continued shear-band propagation is expected to occur after the "stop" event, which indeed can be resolved when correlating both stress and displacement with time, as demonstrated for $198 \mathrm{~K}$ in the inset of Fig. 3. Subsequent to stopping the machine, continued displacement (shear-band creep) can be resolved for a certain amount of time until both stress and displacement remain stationary. We attribute this to a persisting mechanism of shear-band creep prior to arrest. Hence, subsequent to setting the drive of the machine to zero, continued shear-band propagation is detectable for a certain temperature dependent duration; a time scale that we will later show to be of importance for our interpretation. At temperatures below $123 \mathrm{~K}$, a situation is reached, where shear-band arrest remains absent throughout the relaxation-times displayed in Fig. 2. Indeed, when stopping for $500 \mathrm{~s}$ at $78 \mathrm{~K}$ continued shear-band creep of more than $60 \mu \mathrm{m}$ was resolved, until the experiment was resumed ("start") (not shown here).

In conjunction with earlier research establishing the thermal activation of shear banding under strain-rate controlled conditions, ${ }^{10,11}$ the above result of a temperatureindependent stress-relaxation below $123 \mathrm{~K}$ upon stopping suggests a suppression of thermally driven atomic-scale rearrangement processes throughout continued relief of elastically stored energy in the sample-machine assembly. At higher temperatures atomic-scale rearrangement processes quickly allow the shear band to attain arrest under the applied stress during the stopping phase.

Subsequent to the stopping phase of the experiment, stress-overshoots appeared upon reloading ("start"); a feature

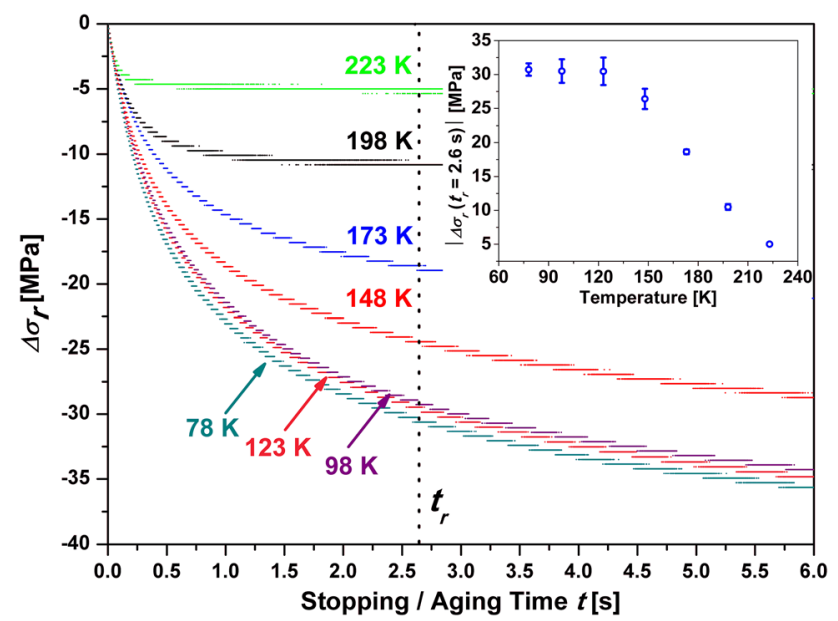

FIG. 2. (Color online) Different stress-relaxation responses as a function of time for various temperatures immediately after stopping the loading experiment. Inset: the absolute stress-relaxation magnitude $\left|\Delta \sigma_{r}\right|$ at an arbitrary time $t_{r}$ of $2.6 \mathrm{~s}$ evidences that the relaxation stress remains constant at temperatures of $123 \mathrm{~K}$ and below. 


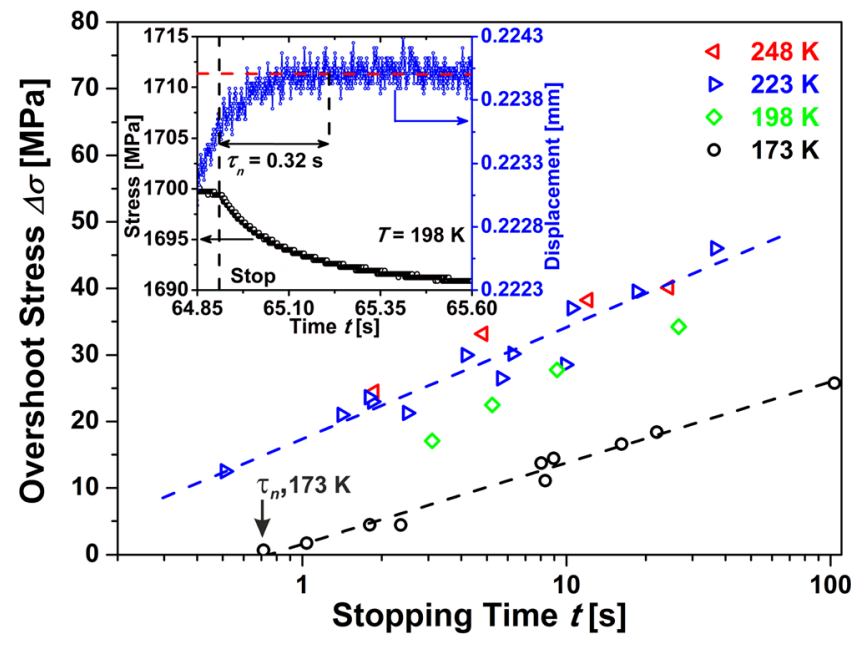

FIG. 3. (Color online) The effect of stopping time on $\Delta \sigma$. Logarithmic fits to the data at each temperature allow the determination of the characteristic time $\tau_{n}$, which increases as a function of decreasing temperature. The inset shows the stress and displacement data as a function of time at $198 \mathrm{~K}$, directly demonstrating shear-band creep subsequent to setting $v_{X H}=0$ (stop), where $\tau_{n}$ corresponds well to the time $(0.32 \mathrm{~s})$ throughout which creep can be resolved prior to attaining shear-band arrest at the horizontal dashed red line.

that was further investigated as a function of time and temperature. In each test, the stopping time $t$ was varied, permitting to plot the stress overshoot magnitude $\Delta \sigma$ as a function of time at different temperatures, as summarized in Fig. 3. It is clear that when choosing a logarithmic time scale, a linear trend of type $\Delta \sigma=C_{1}+C_{2} \log (t)$ becomes apparent. Extrapolating the linear fits to the abscissa allows for the determination of a characteristic time $\tau_{n}$ below which no overshoot occurs, as is exemplarily indicated for $173 \mathrm{~K}$. Thus, when stopping the experiment for shorter durations than $\tau_{n}$, the structural reorganization in the shear-band is insufficient, and upon reapplying a driving force, no measurable excess energy resulting in an overshoot is required. This result is analogous to the time dependency of stiction-spike magnitudes of confined nanoscopic liquids and glassy polymer interfaces, in which this characteristic time is attributed to the time necessary to nucleate a solidlike state from a liquidlike state that prevails during sliding. ${ }^{24-27}$ Note at this stage that by employing a framework known for static friction experiments of nanoscopic confined liquids, we naturally assume the operating shear-band to be a planar defect with strongly reduced viscosity as compared to the surrounding bulk matrix. This assumption is well supported by recent results investigating shear-band properties in bulk metallic glasses. ${ }^{22,30} \mathrm{~A}$ trend of increasing $\tau_{n}$ can be observed as the temperature decreases, as further discussed below and also displayed in Fig. 4. The result in Fig. 3 is consistent with the understanding that $\tau_{n}$ is an inverse measure of the atomicstructure mobility in the confined layer of the shear band. Most importantly, at any temperature, $\tau_{n}$ correlates very well with the time during which continued shear-band creep can be resolved prior to shear-band arrest (inset in Fig. 3).

In summary, stop-and-go experiments thus reveal the following phenomena: The shear band resides first in a dynamic state when it is driven by the applied strain rate. Our results show that when $v_{X H}=0$ ("stop"), a temperature

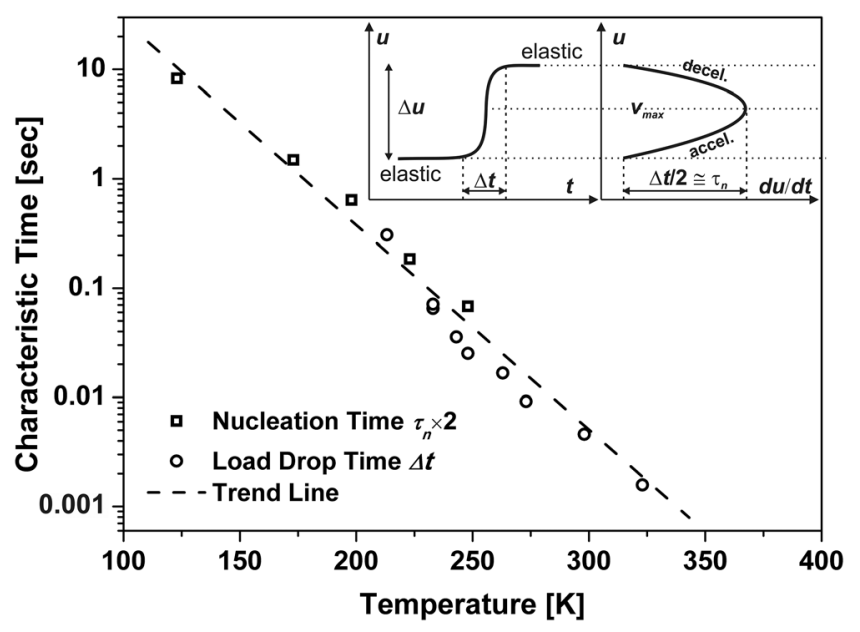

FIG. 4. Characteristic time $\Delta t$ obtained for flow serrations during serrated flow and characteristic time $\tau_{n} \times 2$ as a function of temperature. $\Delta t$ is the load drop time (equivalent to the displacement jump time) during serrated flow, and $\tau_{n}$ the time needed to attain shear-band arrest after stopping during non-serrated flow. Both $\Delta t$ and $\tau_{n} \times 2$ follow the same temperature dependence, indicating that the shear-band deceleration cycle $\Delta t / 2$ is equal to $\tau_{n}$. The dashed line represents a trend line to both data sets. The schematic inset demonstrates the displacement and velocity profiles of a serration as a function of time.

dependent dynamic-to-static transition leads first to continued shear-band creep, followed by arrest and subsequent static aging of the shear band. Further, we observe a very good agreement with $\tau_{n}$ and the shear-band creep duration. We will now proceed with comparing the here-obtained $\tau_{n}$ with the inherent time scales of serrated flow at higher temperatures obtained in our earlier work ${ }^{10,11,13}$ and propose why shear bands arrest by developing the analogy borrowed from static friction experiments of confined liquids. ${ }^{24-27}$ To do this, it is important to point out that the process of a displacement jump during serrated flow has been described by taking into account the time $\Delta t$ over which the increment in displacement $\Delta u$ is created (schematic inset in Fig. 4). ${ }^{10,11,16}$ Differentiating this signal with respect to time allows for plotting the velocity profile for the shear event, which is composed of a symmetric profile (schematic inset in Fig. 4). During the first half, acceleration occurs, followed by a deceleration and arrest of the shear band. Hence, half of the displacement increment time $\Delta t / 2$ is conveyed to represent the same atomic relaxation process during the shear-band creep phase, leading to shear-band arrest, as we observed under non-serrated conditions upon setting $v_{X H}=0$. That means, $\Delta t$ as obtained from serrated flow should obey the same functional dependence as the characteristic time $\tau_{n} \times 2$, if the observed shear-band creep phase is equivalent to the decelerating shear-band propagation cycle. Indeed, this seems to be very much the case and is evidenced in Fig. 4, where the dashed line represents a guide line through all data points.

Based on the good agreement in Fig. 4 and the used analogy from confined liquids, we argue that the question "why do shear bands stop?" finds an answer through the fact that during serrated flow shear-band propagation occurs in a temperature/applied strain-rate regime where the structural freezing of the lower viscous state within the shear band is defined by a very small $\tau_{n}$ relative to the applied strain rate. 
That means, at higher temperatures during serrated flow, $\tau_{n}$ is sufficiently small to quickly reverse the structural softening induced at shear-band initiation, leading to shear-band arrest. At very low temperatures, the stressed shear-band remains in a propagating state since $\tau_{n}$ is so large that thermally driven atomic rearrangement processes do not allow for structural freezing. We may thus interpret the here obtained time $\tau_{n}$ as a characteristic nucleation time for a liquid-like to solid-like transition known to occur in nanometer confined liquids, ${ }^{24,25,31}$ which is in agreement with the fact that the material in the acting shear band undergoes a viscosity change of several orders of magnitude. Theoretical work on glassy systems, which incorporates shear transformation zone dynamics as the fundamental process of plasticity in amorphous alloys, colloidal systems, or granular media, has previously yielded similar stress-overshoot characteristics; ${ }^{32}$ therefore, supporting the experimental route and analogy exploited here. We acknowledge that the effects of structural damage as a function of strain and set-up characteristics on $\tau_{n}$ still compose a challenging continuation of our experimental efforts.

Support by the Swiss National Science Foundation SNF No. 200020-120258 is gratefully acknowledged. R.M. thanks W. L. Johnson for fruitful discussions, as well as the Alexander von Humboldt foundation for financial support during his stay at Caltech.

\footnotetext{
${ }^{1}$ W. Klement, R. H. Willens, and P. Duwez, Nature 187, 869 (1960).

${ }^{2}$ A. Inoue, Acta Mater. 48, 279 (2000).

${ }^{3}$ J. F. Löffler, A. A. Kundig, and F. H. Dalla Torre, in Materials Processing Handbook, edited by J. R. Groza, J. F. Shackelford, E. J. Lavernia, and M. T. Powers (CRC, Boca Raton, 2007), Chap. 17.

${ }^{4}$ W. L. Johnson, MRS Bull. 24, 42 (1999).
}

${ }^{5}$ C. A. Schuh, T. C. Hufnagel, and U. Ramamurty, Acta Mater. 55, 4067 (2007).

${ }^{6}$ M. M. Trexler and N. N. Thadhani, Prog. Mater. Sci. 55, 759 (2010).

${ }^{7}$ M. W. Chen, Annu. Rev. Mater. Res. 38, 445 (2008).

${ }^{8}$ J. Lu, G. Ravichandran, and W. L. Johnson, Acta Mater. 51, 3429 (2003).

${ }^{9}$ M. Heggen, F. Spaepen, and M. Feuerbacher, J. Appl. Phys. 97, 033506 (2005).

${ }^{10}$ D. Klaumuenzer, R. Maass, F. H. D. Torre, and J. F. Löffler, Appl. Phys. Lett. 96, 061901 (2010).

${ }^{11}$ R. Maass, D. Klaumuenzer, and J. F. Löffler, Acta Mater. 59, 3205 (2011).

${ }^{12}$ Y. Q. Cheng, Z. Han, Y. Li, and E. Ma, Phys. Rev. B 80, 134115 (2009).

${ }^{13}$ D. Klaumuenzer, R. Maass, and J. F. Löffler, J. Mater. Res. 26, 1453 (2011).

${ }^{14}$ C. C. Hays, C. P. Kim, and W. L. Johnson, Phys. Rev. Lett. 84, 2901 (2000).

${ }^{15}$ J. R. Greer and J. T. M. De Hosson, Prog. Mater. Sci. 56, 654 (2011).

${ }^{16}$ H. M. Chen, J. C. Huang, S. X. Song, T. G. Nieh, and J. S. C. Jang, Appl. Phys. Lett. 94, 141914 (2009).

${ }^{17}$ B. P. Kanungo, S. C. Glade, P. Asoka-Kumar, and K. M. Flores, Intermetallics 12, 1073 (2004).

${ }^{18}$ P. E. Donovan and W. M. Stobbs, Acta Metall. 29, 1419 (1981).

${ }^{19}$ F. Spaepen, Nature Mater. 5, 7 (2006).

${ }^{20}$ S. Nasuno, A. Kudrolli, A. Bak, and J. P. Gollub, Phys. Rev. E 58, 2161 (1998).

${ }^{21}$ F. H. Dalla Torre, D. Klaumuenzer, R. Maass, and J. F. Löffler, Acta Mater. 58, 3742 (2010).

${ }^{22}$ D. Klaumuenzer, A. Lazarev, R. Maass, F. H. Dalla Torre, A. Vinogradov, and J. F. Löffler, Phys. Rev. Lett. 107, 185502 (2011).

${ }^{23}$ P. F. Guan, M. W. Chen, and T. Egami, Phys. Rev. Lett. 104, 205701 (2010).

${ }^{24}$ J. Klein and E. Kumacheva, Science 269, 816 (1995).

${ }^{25}$ S. Yamada, J. Chem. Phys. 131, 184708 (2009).

${ }^{26}$ T. Baumberger and C. Caroli, Adv. Phys. 55, 279 (2006).

${ }^{27}$ H. Yoshizawa and J. Israelachvili, J. Phys. Chem. 97, 11300 (1993).

${ }^{28}$ Y. Kawamura, T. Shibata, A. Inoue, and T. Masumoto, Appl. Phys. Lett. 69, 1208 (1996).

${ }^{29}$ R. Maass, D. Klaumuenzer, E. I. Preiss, P. M. Derlet, and J. F. Löffler, Scr. Mater. 66, 231 (2012).

${ }^{30}$ J. Bokeloh, S. V. Divinski, G. Reglitz, and G. Wilde, Phys. Rev. Lett. 107, 235503 (2011).

${ }^{31}$ P. A. Thompson and M. O. Robbins, Science 250, 792 (1990).

${ }^{32}$ A. Lemaitre and J. Carlson, Phys. Rev. E 69, 061611 (2004). 